\title{
Co-movement of COVID-19 and B̈itcoin: Evidence from wavelet coherence analysis
}

\author{
John W. GOODELL* \\ University of Akron Department of Finance, Akron, Ohio, United States \\ Stephane GOUTTE \\ Université Paris-Saclay, UVSQ, CEMOTEV, 78280, Guyancourt, France
}

May 19, 2020

\begin{abstract}
We apply wavelet methods to daily data of COVID-19 world deaths and daily Bitcoin prices from 31th December 2019 to 29th April 2020. We find, especially for the period post April 5, that levels of COVID-19 caused a rise in Bitcoin prices. We contribute to the fast-growing body of work on the financial impacts of COVID-19, as well as to ongoing consideration of whether Bitcoin is a safe haven investment. Our results should be of great interest to both scholars and policy makers, as well as investment professionals interested in the financial implications of both COVID-19 and cryptocurrencies.
\end{abstract}

Keywords: Co-movement; COVID-19; Bitcoin; Wavelet; Safe haven JEL classification: C58; F37; G14; G15; Q31

\section{Introduction and motivations}

At the time of writing this article, the COVID-19 pandemic is still ongoing, with the eventual scale of the disaster not yet determined. We are all now tragically familiar with the ongoing enormous costs in lives of this pandemic. However, we are also concerned about the eventual economic impact of this crisis, including the impact on financial markets. In response to this, we investigate the associate of Bitcoin.

We apply the wavelet method of Grinsted et al. (2004) (also used by others, e.g., Kang et al., 2019) to daily data of COVID-19 world deaths and daily Bitcoin prices from 31th December 2019 to 29th April 2020. With this wavelet

*Corresponding author: johngoo@uakron.edu 
approach we find that levels of COVID-19 caused a rise in Bitcoin prices. This effect is especially strong for the portion of our period post April 5 . We consider that our study contributes to the rapidly growing body of work on the financial impacts of COVID-19, as well as to an ongoing focus in the literature regarding whether Bitcoin is a safe haven investment.

Our results are especially meaningful when considering that as COVID-19 unfolds there is greater concern for future economic costs, and likely greater economic uncertainty. As noted by Goodell (2020), the ongoing COVID-19 crisis will have enormous ongoing economic costs. Prior to the current crisis, for instance, Bloom, Cadarette, and Sevilla (2018) point out the economic vulnerability posed by potential pandemics, both in terms of costs to the health system, as well as costs of social distancing: loss to employment productivity, loss to consumer demand, an impact on tourism and other particular industries, and an impact on foreign direct investment ${ }^{1}$. This concern is echoed by Fan, Jamison, and Summers (2018). They note very recently "an unmet need for greater investment in preparedness against major epidemics and pandemics." They estimate the expected annual losses from pandemic risk to be annually approximately 500 billion US dollars, or $0.6 \%$ of global income. Considering the costs of COVID-19, this seemingly large sum now seems greatly underestimated. Other works highlighting the need for economic risk management vis-à-vis the potential likelihood of future pandemics include Bloom and Canning (2004); Cavallo et al. (2013); Lewis (2001); Madhav et al. (2017); Tam, Khan, and Legido-Quigley (2016); Yach, Stuckler, and Brownell (2006) and many others. Lagoarde-Segot and Leoni (2013) model the stress to the banking industries of developing countries as a result of large pandemics.

COVID-19 could also lead to a very long-term shifting in costs of equity. As noted by Goodell (2020), Lee and McKibbin (2004) find a 200-basis point increase in the country risk premium for China and Hong Kong following SARS. However, China and Hong Kong were particular risk areas for SARS, while COVID-19 is global. Consequently, a large increase in perceived equity risk is reasonably expected.

We consider our study alongside a number of recent papers that investigate the hedging properties of cryptocurrencies. As early as Dyhrberg (2016a) and Dyhrberg (2016b), it has been considered that Bitcoin might share some of the same hedging properties as gold (Baur and Lucey, 2010; Baur and McDermott, 2010; Bredin, Conlon, and Potì, 2015; O'Connor et al., 2015; Raza, Shah, and Shahbaz, 2018). As with many of the aspects of Bitcoin, our understanding is still developing. It is by no means a settled issue that Bitcoin is a new gold (Klein, Pham Thu, and Walther, 2018) ${ }^{2}$.

As gold is often considered to move with economic policy uncertainty (Raza, Shah, and Shahbaz, 2018), it is natural to extend this inquiry to Bitcoin. Cer-

\footnotetext{
${ }^{1}$ See also Goodell and Huynh (2020) for impact of COVID-19 on US industries

${ }^{2}$ One hopes perhaps, that the mechanism of any negative correlation between economic conditions and Bitcoin is not simply due to economic downturns causing increased portions of economic output being in illegal or shadow activities that are financed by Bitcoin (Foley, Karlsen, and Putniņš, 2019).
} 
tainly, there are numerous studies investigating the co-movement of Bitcoin and similar currencies with movement in global economic uncertainty. Again, this area is not settled. For instance, Wu et al. (2019) and Wang et al. (2019) find a negligible relationship, while Bouri et al. (2018) and Demir et al. (2018) find a stronger relationship.

It is also reasonable to consider further the movement of Bitcoin with other assets, particularly in the context of changes in economic apprehension. After all, economic conditions manifest to financial markets through changes in asset prices. While Bouri et al. (2017b), Klein et al. (2018) and Smales (2018) do not find consistent evidence that Bitcoin serves as a safe haven for global assets, Selmi et al. (2018) show that Bitcoin, like gold, serves as a hedge, safe haven, and diversifier for oil price movements. However, this property seems to be sensitive to the Bitcoin's and gold's different (bear, normal or bull) market conditions and to whether the oil price is in a downside, normal or upside regime. Kurka (2019) find that the relationship between Bitcoin and other assets depends on whether there are shocks occurring. Further, Matkovskyy and Jalan (2019) find that, during crisis periods, risk-averse investors tend to move away from Bitcoin, with a view that it is riskier than financial markets.

A further question is how the role of Bitcoin in hedging other assets has changed during the period of the COVID-19 crisis. Understandably there are few studies yet on such recent events. An exception is Conlon and McGee (2020), who show that Bitcoin has indeed been a poor hedge against the SP500 during the COVID-19 crisis.

Overall, our study of how Bitcoin prices co-move with the intensity of the COVID 19 crisis will contribute considerably to our understanding both of how COVID-19 is impacting financial systems; as well as how Bitcoin might act as a hedge, diversifier, or safe haven during extreme, global, crises.

\section{Data and econometric framework}

This study applies the wavelet method of Grinsted et al. (2004) to daily data of COVID-19 world deaths and Bitcoin prices from 31th December 2019 to 29th April 2020.

\subsection{Econometric framework}

In contrast to standard time series modelling, the wavelet coherence approach allows capturing of the co-movement between two time series in both the time and frequency domains. The wavelet approach uses a bivariate framework established on a continuous wavelet transform ( Morlet set to 6). This allows for a variety of scaled localizations (Rua and Nunes, 2009). In order to capture and interpret co-movement between time series both in time and frequency do-

mains, we suggest the use of the wavelet coherence approach by using both the cross-wavelet transform and coherence. 
Following the approach of Torrence and Compo (1998), the cross wavelet transform of two time-series $x(t)$ and $y(t)$ is defined using their own cross wavelet transfrom $(\mathrm{CWT}) W_{n}^{x}(u, s)$ and $W_{n}^{y}(u, s)$ as:

$$
W_{n}^{x y}(u, s)=W_{n}^{x}(u, s) * W_{n}^{y}(u, s)
$$

where $u$ is associated to the location and $s$ to the scale. The $*$ denotes the complex conjugate. The cross wavelet transform highlights areas in the time-frequency domain where time series show a high common power. In other words, it captures the local covariance between the two time series $x(t)$ and $y(t)$ at each scale.

After Torrence and Webster (1999), we define the wavelet coherence capturing the co-movement between two time series as:

$$
R^{2}(u, s)=\frac{\left|S\left(s^{-1} W^{x y}(u, s)\right)\right|^{2}}{S\left(s^{-1}\left|W^{x}(u, s)\right|^{2}\right) S\left(s^{-1}\left|W^{y}(u, s)\right|^{2}\right)}
$$

where $S$ is a smoothing operator over time as well as scale and $0 \leq R^{2}(u, s) \leq$ 1 (Rua and Nunes, 2009). The quantity $R^{2}(u, s)$ represents the wavelet squared coherence. Its value is between 0 and 1 . The higher its value, the higher the co-movement between the two time series. We note that, unlike the classical correlation of two time series, wavelet squared coherence is restricted to positive values. Therefore, that at this step this methodology can not distinguish positive or negative co-movement, or between positive and negative correlation. A way to solve this problem is to use the phase difference of Terrence and Compo (1998) to capture the two possible co-movements: positive and negative. Moreover, in the spirit of Granger causality testing, this graphical presentation of the wavelet squared coherence can give us the causal relationships between the two time series.

Wavelet coherence phase difference is defined as:

$$
\Phi_{x y}(u, s)=\tan ^{-1}\left(\frac{\operatorname{Im}\left\{S\left(s^{-1} W^{x y}(u, s)\right)\right\}}{\operatorname{Re}\left\{S\left(s^{-1} W^{x y}(u, s)\right)\right\}}\right)
$$

where, Im and $R e$ are the imaginary and real parts of the smoothed crosswavelet transform, respectively.

Phases are indicated by black arrows on the wavelet coherence plots. A phase-difference of zero is consistent with time series moving together. Arrows pointing to the right (left) indicate time series which are in-phase (out of phase), or are positively (negatively) correlated. An arrow pointing upward indicates that the first time series leads the second by $\pi / 2$; whereas a downward pointing arrow pointing suggests the second time series is leading the first by $\pi / 2$. A combination of positions is usual.

We compute the daily returns by finding the difference between two consecutive prices: $r i, t=P_{i, t}-P_{i, t 1}$ with $i$ is for COVID-19 deaths and Bitcoin.

Figure 2.1 shows the dynamics of the return series and illustrates the stylized factors (e.g., volatility clustering) for the Bitcoin return series. 


\section{Deaths}

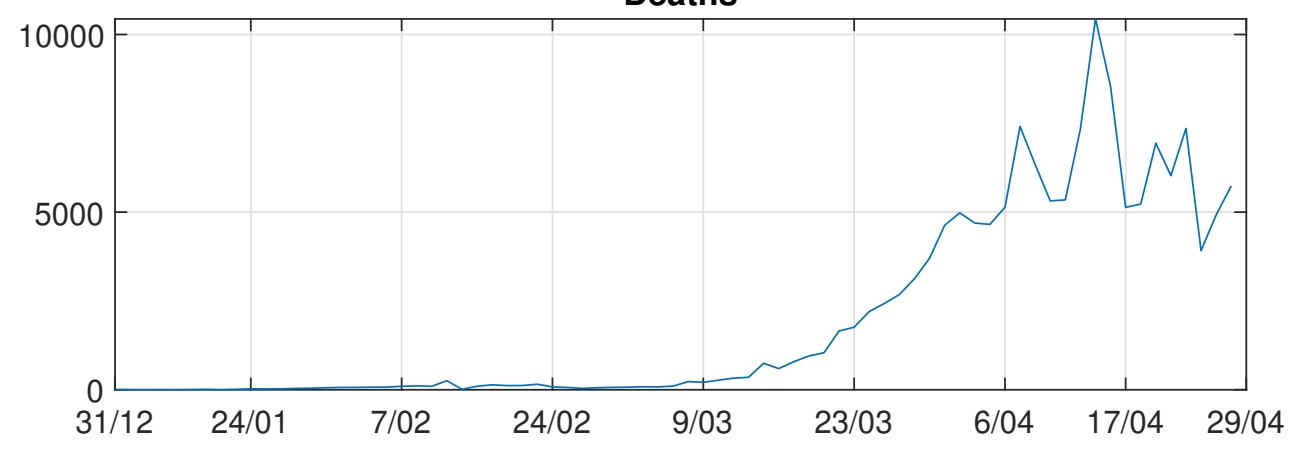

Bitcoin

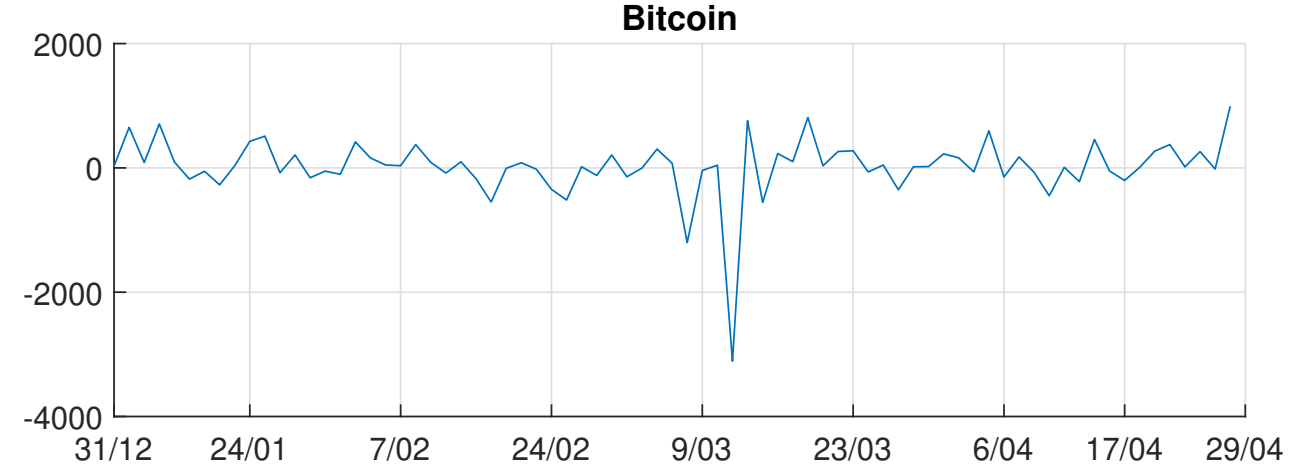

Figure 1: Evolution dynamics of the return series 
We see on Figure 2.1, that the dynamic of the COVID-19 pandemic is a twophase processe: an exponential increasing of the death's number until middle of April then a quite linear decreasing.

Table 1 presents descriptive statistics of the return series. booktabs booktabs

Table 1: Descriptive Statistics

$\begin{array}{lrr} & \text { Covid Deaths } & \text { Bitcoin } \\ \text { Mean } & 1889.519 & 20.602 \\ \text { Std. Deviation } & 2678.431 & 488.075 \\ \text { Skewness } & 1.272 & -3.507 \\ \text { Std. Error of Skewness } & 0.274 & 0.274 \\ \text { Kurtosis } & 0.491 & 22.184 \\ \text { Std. Error of Kurtosis } & 0.541 & 0.541 \\ \text { Shapiro-Wilk } & 0.730 & 0.704 \\ \text { P-value of Shapiro-Wilk } & <.001 & <.001\end{array}$

As shown in Table 1, Bitcoin exhibits skewing, as well as excess kurtosis. These findings and the Shapiro-Wilk statistics demonstrate that the distributions of this series is asymmetric and leptokurtic, rejecting the normality property.

\subsection{Wavelet coherence}

We apply continuous wavelet power spectrum and coherence methodologies between COVID-19 deaths and Bitcoin return prices. This is illustrated in Figures 2.2 and 2.2. The wavelet power spectrum is defined as the absolute value of the square of the wavelet transform. It gives us a measure of the time series variance for each time and for each scale (frequency). The horizontal axis displays the time component, while the vertical axis shows the frequency component. This ranges from scale 1 (a day) up to scale 16 (more than 16 days). The black contours in the plot indicate regions with significance at the five-percent level. This is estimated by Monte Carlo simulation with phase-randomized surrogate series. The solid curved line shows the cone of influence, or the zone affected by edge effects. 

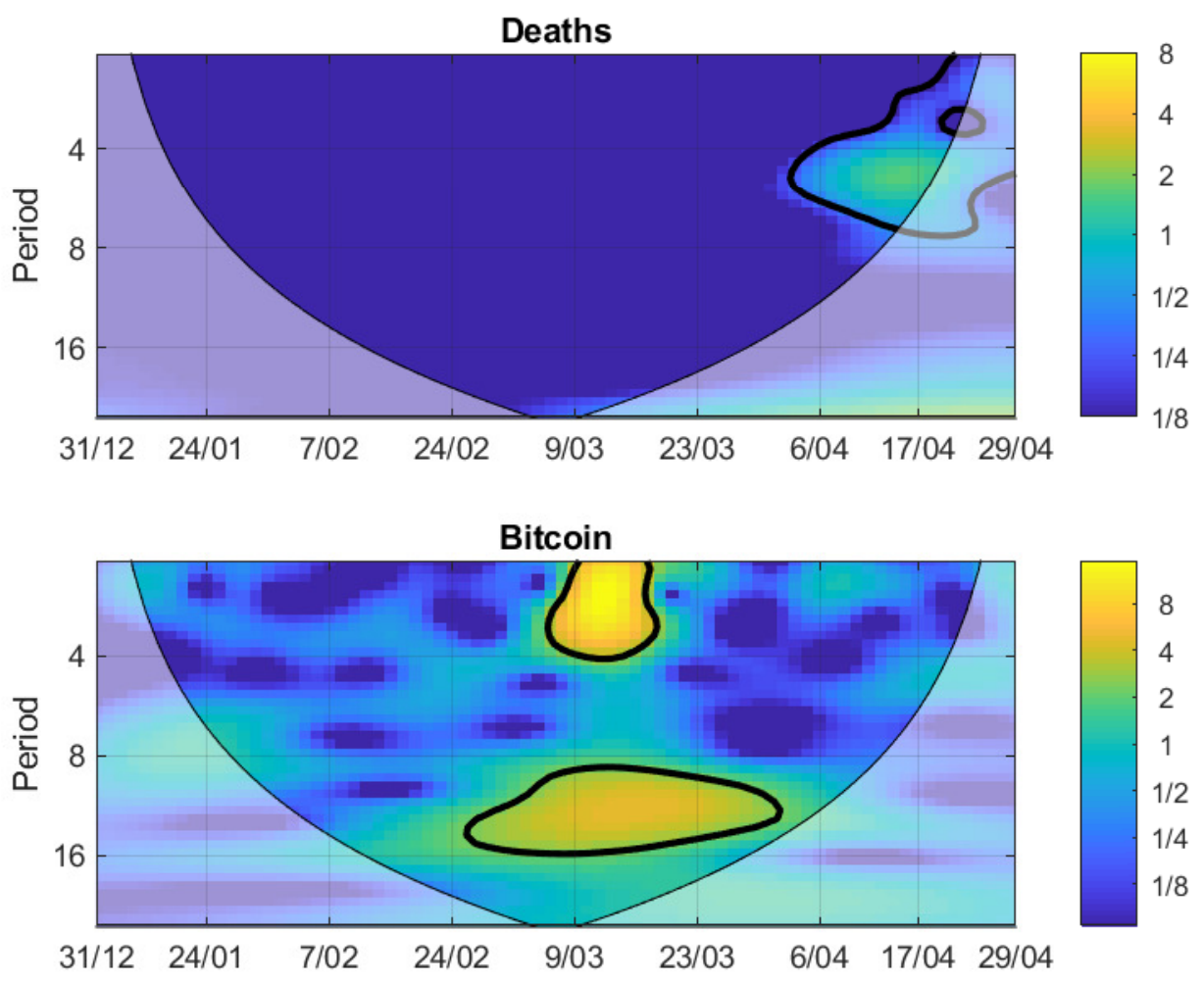

Figure 2: Continuous wavelet transform of COVID-19 deaths and Bitcoin returns. The horizontal axis shows time, while the vertical axis refers to the period in days.. 


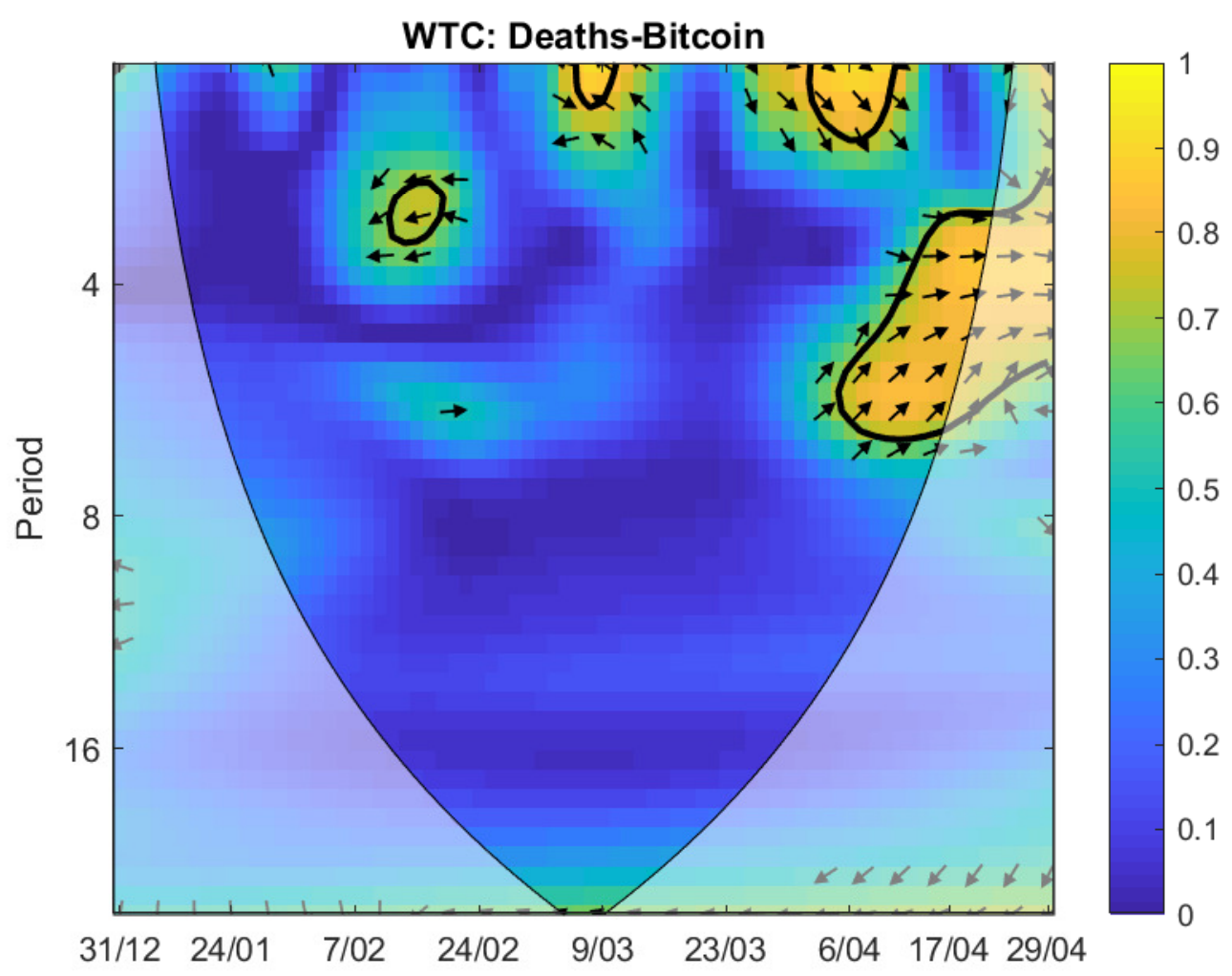

Figure 3: Wavelet coherency (WTC) between COVID-19 deaths and Bitcoin. The level of correlation is indicated by the color on the right side of the charts; the hotter the color (moving from cool (blue) to hot (yellow)) the higher the absolute correlation value with respect to $R^{2}(u, s)$. 
Figure 2.2 illustrates the wavelet coherence and phase difference between COVID-19 deaths and Bitcoin return prices. The coherency ranges from yellow (high coherency) to blue (low coherency) to designate the amount of comovement. Therefore, a yellow color represents strong co-movement; whereas a blue color indicates weak co-movements. We van identify four significant high-degree co-movements between COVID-19 deaths and Bitcoin.

- For the 10th February to 20th February period across the 2-4 day frequency band.

- A two-day frequency cycle observed during the period 8th March to 10th March and another one during the period 5th April to 10th April.

- A particularly meaningful and significant coherence for 3-7 day frequency cycles observed during the period 5th April to 29th April.

These coherences evidence the contagion effect during the implementation of containment in the majority of the countries of the world (i.e. after middle of March). The increasing of COVID-19 deaths was associated with a reduced value of Bitcoin for short and mid terms during this period. Perhaps these periods roughly match 1) the beginning of the pandemic (i.e., a global threat outside of China; 2) the beginning of large-scale enforced social distancing in many Western countries; and 3) the full global manifestation of COVID-19.

Additionally, we discern causality and phase differences between COVID19 deaths and Bitcoin returns. Arrows indicate the phase differences between COVID-19 deaths and Bitcoin returns. For example, $\rightarrow$ and $\leftarrow$ indicate that both COVID-19 deaths and Bitcoin returns are in phase and out of phase. Being in phase (out of phase) indicates a positive (negative) correlation between COVID-19 deaths and Bitcoin returns. Moreover, $\nearrow$ and $\swarrow$ indicate that COVID-19 deaths are leading Bitcoin return prices, while $\searrow$ and $\nwarrow$ indicate COVID-19 deaths values are lagging those of Bitcoin return prices.

Thus as shown in Figure 2.2, we can identify causality and phase differences between COVID-19 deaths and Bitcoin return prices. We observe that since 5th April, the main and most significant period of coherence and co-movements in both 1-2 days and 3-7 day bands, that arrows are in majority $\nearrow$ and $\rightarrow$, signifying an in-phase relationship and a positive correlation between COVID-19 deaths and Bitcoin returns. Moreover, these arrows also indicate that COVID19 deaths led Bitcoin returns in the short and medium terms, over this period of full world containment.

In summary, we find predominantly a strong negative co-movement of Bitcoin prices and COVID-19. However, levels of the intensity of this co-movement do vary over our overall period of study, with some brief intervals even exhibiting a positive association. It is interesting to compare our results with Demir et al. (2018). Demir et al. (2018) find a negative association of economic policy uncertainty with Bitcoin only for extreme values of this uncertainty. Certainly COVID-19 is an extreme event, with likely an extreme impact on peoples' perception of uncertainty. Our results may result in more questions than answers for future research. For instance, how does the co-movement of Bitcoin 
with COVID-19 compare with the co-movements of national financial markets with the pandemic? Why were there strong but brief periods of negative comovement of Bitcoin with COVID-19 as early as February 10? Could Bitcoin have a signaling value? Many of these questions are outside the bounds of this paper.

\section{Conclusions}

As the COVID-19 pandemic continues, with the eventual scale of the disaster not yet determined, we are all painfully aware of the continuing cost in lives of this pandemic. However, there is also concern about the eventual economic impact of this crisis, including the effect on financial markets. Additionally, academic finance has also had, in the last few years, considerable interest in the hedging, diversifying and safe-haven qualities of Bitcoin. In response to this, we investigate the co-movement of Bitcoin with levels of COVID-19 related fatalities. We consider that while national equity markets reflect economic concerns at country levels, the evolving COVID-19 crisis affords a measure of a developing global economic threat. We apply wavelet methods to daily data of COVID-19 world deaths and daily Bitcoin prices from 31th December 2019 to 29th April 2020, evidencing that levels of COVID-19 cause a rise in Bitcoin prices. This effect is especially strong for the portion of our period post April 5. We consider that our study contributes both to the fast-growing body of work on the financial impacts of COVID-19; as well as to an ongoing focus in the literature regarding whether Bitcoin is a safe haven investment.

\section{References}

Baur, Dirk G., and Brian M. Lucey, (2010), "Is Gold a Hedge or a Safe Haven? An Analysis of Stocks, Bonds and Gold", Financial Review 45 (2) pp. 217-229.

Baur, Dirk G., and Thomas K. McDermott, (2010), "Is gold a safe haven? International evidence", Journal of Banking and Finance 34 (8) pp. 1886-1898.

Bloom, David E, and David Canning, (2004), "Epidemics and economics: Interactions between global change and human health ", Scripta Varia 106 pp. 304-331.

Bloom, David E. , Daniel Cadarette, and JP Sevilla, (2018), "Epidemics and economics: New and resurgent infectious diseases can have far-reaching economic repercussions", Finance and Development 55 (2) pp. 46-49.

Bouri, Elie, Rangan Gupta, Chi Keung Marco Lau, David Roubaud, and Shixuan Wang, (2018), "Bitcoin and global financial stress: A copula-based approach to dependence and causality in the quantiles", Quarterly Review of Economics and Finance 69 pp. 297-307. 
Bredin, Don, Thomas Conlon, and Valerio Potì, (2015), "Does gold glitter in the long-run? Gold as a hedge and safe haven across time and investment horizon", International Review of Financial Analysis 41 pp. 320-328.

Cavallo, Eduardo, Sebastian Galiani, Ilan Noy, and Juan Pantano, (2013), "Catastrophic natural disasters and economic growth", Review of Economics and Statistics 95 (5) pp. 1549-1561.

Conlon, Thomas, and Richard J. McGee, (2020), "Safe haven or risky hazard? Bitcoin during the COVID-19 bear market", Finance Research Letters forthcoming.

Demir, Ender, Giray Gozgor, Chi Keung Marco Lau, and Samuel A. Vigne, (2018), "Does economic policy uncertainty predict the Bitcoin returns? An empirical investigation", Finance Research Letters 26 pp. 145-149.

Dyhrberg, Anne Haubo, (2016a), "Bitcoin, gold and the dollar - A GARCH volatility analysis", Finance Research Letters 16 pp. 85-92.

Dyhrberg, Anne Haubo, (2016b), "Hedging capabilities of bitcoin. Is it the virtual gold?", Finance Research Letters 16 pp. 139-144.

Fan, Victoria Y., Dean T. Jamison, and Lawrence H. Summers, (2018), "Pandemic risk: how large are the expected losses?", Bulletin of the World Health Organization 96 (2) pp. 129-134.

Foley, Sean, Jonathan R Karlsen, and Tālis J Putniņš, (2019), "Sex, drugs, and bitcoin: How much illegal activity is financed through cryptocurrencies?", Review of Financial Studies 32 (5) pp. 1798-1853.

Goodell, John W, (2020), "COVID-19 and finance: Agendas for future research", Finance Research Letters forthcoming.

Goodell, John W., and Toan Luu Duc Huynh, (2020), "Did Congress trade ahead? Considering the reaction of US industries to COVID-19 ", Finance Research Letters forthcoming.

Grinsted, A., J. C. Moore, S. Jevrejeva (2004), "Application of the cross wavelet transform and wavelet coherence to geophysical time series", Nonlinear Processes in Geophysics, 11.

Sang Hoon Kang, Ron P. McIver, Jose Arreola Hernandez, (2019), "Co-movements between Bitcoin and Gold: A wavelet coherence analysis", Physica A 536..

Klein, Tony, Hien Pham Thu, and Thomas Walther, (2018), "Bitcoin is not the New Gold - A comparison of volatility, correlation, and portfolio performance", International Review of Financial Analysis 59 pp. 105-116.

Kurka, Josef, (2019), "Do cryptocurrencies and traditional asset classes influence each other?", Finance Research Letters 31 pp. 38-46.

Lagoarde-Segot, Thomas, and Patrick L Leoni, (2013), "Pandemics of the poor and banking stability", Journal of Banking and Finance 37 (11) pp. 4574-4583. 
Lee, Jong-Wha, and Warwick J McKibbin, (2004), "Estimating the global economic costs of SARS", Learning from SARS: preparing for the next disease outbreak: workshop summary (National Academies Press Washington, DC).

Lewis, Maureen, (2001), "The Economics of Epidemics", Georgetown Journal of International Affairs 2 pp. 25.

Madhav, Nita, Ben Oppenheim, Mark Gallivan, Prime Mulembakani, Edward Rubin, and Nathan Wolfe, (2017), "Pandemics: risks, impacts, and mitigation", in Disease Control Priorities: Improving Health and Reducing Poverty. 3rd edition The International Bank for Reconstruction and Development/The World Bank)

Matkovskyy, Roman, and Akanksha Jalan, (2019), "From financial markets to Bitcoin markets: A fresh look at the contagion effect", Finance Research Letters 31 pp. 93-97.

O'Connor, Fergal A., Brian M. Lucey, Jonathan A. Batten, and Dirk G. Baur, (2015), "The financial economics of gold - A survey", International Review of Financial Analysis 41 pp. 186-205.

Raza, Syed Ali, Nida Shah, and Muhammad Shahbaz, (2018), "Does economic policy uncertainty influence gold prices? Evidence from a nonparametric causality-in-quantiles approach", Resources Policy 57 pp. 61-68.

A. Rua, L.C. Nunes, (2009), "International co-movement of stock returns: A wavelet analysis", Journal of Empirical Finance 11 pp. 632-639.

Selmi, Refk, Walid Mensi, Shawkat Hammoudeh, and Jamal Bouoiyour, (2018), "Is Bitcoin a hedge, a safe haven or a diversifier for oil price movements? A comparison with gold", Energy Economics 74 pp. 787-801.

Smales, Lee A, (2019), "Bitcoin as a safe haven: Is it even worth considering?", Finance Research Letters 30 pp. 385-393.

Tam, Clarence C, Mishal S Khan, and Helena Legido-Quigley, (2016), "Where economics and epidemics collide: Migrant workers and emerging infections", The Lancet 388 (10052) pp. 1374-1376.

C. Torrence, G.P. Compo, (1998), "A practical guide to wavelet analysis", Bulletin of the American Meteorological Society 79 pp. 61-78.

C. Torrence, P.J. Webster, (1999), "Interdecadal changes in the enso-monsoon system", Journal of Climate 12 pp. 2679-2690.

Wang, Gang-Jin, Chi Xie, Danyan Wen, and Longfeng Zhao, (2019), "When Bitcoin meets economic policy uncertainty (EPU): Measuring risk spillover effect from EPU to Bitcoin", Finance Research Letters 31 pp. 489-497.

Wu, Shan, Mu Tong, Zhongyi Yang, and Abdelkader Derbali, (2019), "Does gold or Bitcoin hedge economic policy uncertainty?", Finance Research Letters 31 pp. 171-178. Yach, Derek, David Stuckler, and Kelly D Brownell, (2006), "Epidemiologic and economic consequences of the global epidemics of obesity and diabetes", Nature Medicine 12 (1) pp. 62-66. 